\title{
A Comparative Study of the Mitochondrial Structure of Petite Strains of Saccharomyces cerevisiae
}

\author{
By D. G. SMITH, R. MARCHANT, N. G. MAROUDAS \\ AND D. WILKIE \\ Department of Botany, University College, London, W.C. I
}

(Accepted for publication 27 November 1968)

\section{SUMMARY}

\begin{abstract}
A respiratory-competent strain of Saccharomyces cerevisiae (ID) had mitochondria of diverse morphology. A spontaneous petite strain and petites induced by acriflavine, ultraviolet-radiation or phenethyl alcohol showed similar mitochondrial diversity to the parent strain, but in all the petites except the spontaneous one there were fewer mitochondrial profiles showing cristae. In another respiratory-competent strain (I I) the mitochondria were of uniform morphology. A phenethyl alcohol-induced petite derived from this strain again showed fewer cristate mitochondrial profiles. There was no evidence of the grossly aberrant and lamellate mitochondrial profiles observed in strain $\mathrm{I} D$ and its petites. Each of the cultures obtained from an ascospore tetrad from a I D $\times$ I I cross was induced to form petites with phenethyl alcohol; all showed a significant reduction in the proportion of mitochondrial profiles with cristae. Two of them showed the mitochondrial diversity characteristic of parent strain I D. Other features of mitochondrial morphology appear to be under nuclear control and are strain-dependent. All the petites were cytoplasmic mutants and lacked cytochromes $a$ and $b$.
\end{abstract}

\section{INTRODUCTION}

In certain facultatively anaerobic yeasts, including Saccharomyces cerevisiae, a group of mutants may be produced, known as 'petites' (Ephrussi, Hottinguer \& Chimenes, 1949). These mutants have defective mitochondria and are therefore unable to grow on non-fermentable substrates. A characteristic of petite yeasts is the absence of cytochrome $a$ and usually also of cytochrome $b$. The petite condition is normally inherited cytoplasmically ( $\rho$-petites), but also arises by mutation of nuclear genes ( $p$-petites). Glucose represses the formation of mitochondrial cristae in respiratory-competent yeast (see Marchant \& Smith, 1968), and also of cytochromes $a$ and $b$ (see Roodyn \& Wilkie, I968).

Isolated mitochondria have DNA, ribosomal and transfer RNA, and are able to incorporate amino acids into an insoluble protein fraction. However, the exact relationship between mitochondrial DNA and mitochondrial structure remains unclear. It has not yet been established that mitochondrial DNA is directly involved in specifying mitochondrial protein. The morphology of mitochondria, even in $\rho$-cells, could still be largely dictated by nuclear genes. The petite condition can be induced by a wide range of mutagens, and the question arises whether these mutagens result in the same $\rho$ - phenotype, even in yeasts of different genotype.

An initial investigation, by Yotsuyanagi (1962), of acriflavine-induced $\rho-$ petites, 
revealed structural alterations in the mitochondria, whereas $p$ petites had mitochondria similar to those found in respiratory-competent yeasts. Both categories of petite lacked cytochrome $a$. The yeasts were grown in $4 \%$ glucose medium; during the early phase of growth mitochondria were few in number and had poorly developed cristae, presumably because of glucose repression. Yeasts from late stationary phase had larger mitochondria, often containing lamellations of the inner membrane. A major difference between $\rho+$ and $\rho$ - cultures was that the former alone possessed welldefined cristate mitochondria throughout late log. phase.

Avers, Pfeffer \& Rancourt (I965) have also examined acriflavine-induced petites of Saccharomyces cerevisiae from structural and histochemical viewpoints. Instead of cytochrome $a$ spectra, cytochrome oxidase activity was measured. Using a medium with $\mathrm{I} \%$ glucose, they found that mitochondria in petites completely lacking in cytochrome oxidase were similar to those described by Yotsuyanagi (I962) from logarithmic phase $\rho$ - cultures: i.e. the mitochondria were non-cristate. On the other hand, yeasts in which cytochrome oxidase activity was low, appeared to have mitochondria indistinguishable from the wild type (Avers, Pfeffer \& Rancourt, 1965; Avers, Rancourt \& Lin, 1965).

Petites of Saccharomyces cerevisiae produced by $p$-nitrophenol treatment, grown under unspecified conditions on glucose, showed few and lamellate mitochondria (Osumi \& Katoh, 1967).

Bowers, McClary \& Ogur (1967) have demonstrated the importance of glucose repression in these studies on mitochondrial structure. A petite strain, of unspecified origin, grown on $2 \%$ glucose had non-cristate mitochondrial profiles. The same mutant grown in a chemostat with a limiting low concentration of glucose showed mitochondria indistinguishable from the wild type.

In the present study glucose repression was avoided by using melibiose, a fermentable but non-repressing substrate (Reilly \& Sherman, 1965). We have compared petites induced by various mutagens on a single strain, and by one mutagen (phenethyl alcohol) on a set comprising two parental strains and their resultant tetrad progeny. We have looked for the mitochondrial peculiarities reported in petite yeasts by other workers, i.e. lamellate cristae or lack of cristae, and by counting profiles, have examined whether these features are consistently more frequent in a range of petites. These features were also examined for evidence of nuclear, as opposed to cytoplasmic, inheritance.

\section{METHODS}

Yeast strains. Haploid strains I I and I D of Saccharomyces cerevisiae were crossed on minimal medium. The resultant diploid cells were sporulated, and ascospores dissected with a de Fonbrune micromanipulator. One tetrad, 2 A-D, was selected for the present investigation. Mating type and growth requirements were as follows:

$$
\begin{array}{ll}
\text { Parents: } & \text { I D-a } / a d, \text { leu, lys } \\
& \text { I I }-\alpha / a d_{2} \\
\text { Progeny: } & 2 \mathrm{~A}-\mathrm{a} / \text { leu, lys } \\
& 2 \mathrm{~B}-\alpha / a d, \text { leu, lys } \\
& 2 \mathrm{C}-\mathrm{a} / a d_{2} \\
& 2 \mathrm{D}-\alpha / a d
\end{array}
$$


Cultural conditions. The following media were used. YEPS: I \% Difco yeast extract, $2 \%$ Difco Bacto-peptone, $2 \%$ glucose. YEPM, YEPG: as for YEPS but with $2 \%$ melibiose or $4 \%$ glycerol as carbon source. PDM: petite differential medium, YEPG with addition of $0.2 \%$ glucose. MMS: minimal medium of Wickerham (1946), containing salts, vitamins and glucose only. SPM: sporulating medium of $0.3 \%$ raffinose, $0.2 \%$ sodium acetate. Media were solidified with $2 \%$ agar; incubation temperature was $30^{\circ}$.

Liquid cultures were shaken at $30^{\circ}$ in $250 \mathrm{ml}$. flasks containing $100 \mathrm{ml}$. melibiose medium. The yeasts were harvested in late log. phase: $24 \mathrm{hr}$ for $\rho+$ cells, $48 \mathrm{hr}$ for $\rho-$.

Petites. Petite colonies were recognized by their small size on PDM. To distinguish true petites from the small, slow-growing, respiratory-competent colonies which arose after treatment with ultraviolet radiation or phenethyl alcohol (PEA), suspected petite colonies from the PDM plates were suspended in water and dropped on to YEPG plates. Only those clones which showed no signs of growth after a week on the glycerol plates were selected for further examination. Suspected $\rho$ - strains were confirmed by crossing with a $\rho+$ strain, where the resulting zygotes were respiratory-competent and sporulated to give all $\rho+$ progeny, and by crossing with another $\rho$ - strain where the zygotes were not respiratory-competent and did not sporulate.

Spontaneous petites, comprising about $2 \%$ of the population, were isolated from melibiose plates.

Ultraviolet-induced petites were obtained by exposing plated yeasts, at a density of $200 /$ plate, for I min. to a Phillips $6 \mathrm{~W}$ TUV lamp, at $\mathrm{I0} \mathrm{cm}$. The kill was about $80 \%$, with $20 \%$ petites among the survivors.

Acriflavine petites were induced by adding acriflavine $5 \mu \mathrm{g}$. $/ \mathrm{ml}$. to YEPS medium, giving $100 \%$ induction.

PEA petites were obtained from colonies dropped out on to YEPS plates containing $0.1 \%$ phenethyl alcohol (Eastman Chemical Co.). The population was found to vary from $90 \% \rho-$ to $90 \% \rho+$, depending on the strain.

Cytochrome determination. Yeasts grown in melibiose medium were harvested and washed twice in $2 \%$ sodium dithionite solution, to reduce the cytochromes. The yeasts were resuspended in an equal volume of the dithionite solution and placed in the I cm. cuvette of a Unicam SP 800 split-beam recording spectrophotometer. Spectra were examined at room temperature, against a blank consisting of several sheets of filter paper; this blank had no absorption peaks in the 300 to $700 \mathrm{~m} \mu$ range.

Electron microscopy. Yeasts from the end of the logarithmic growth phase were harvested, washed twice in distilled water and then fixed in $\mathrm{I} \cdot 5 \%$ aqueous $\mathrm{KMnO}_{4}$ for $4 \mathrm{hr}$ at room temperature. Permanganate was the preferred fixative in this study as it gives reasonable fixation of membranous structures in intact Saccharomyces cerevisiae. After fixation and washing, the yeasts were centrifuged and left in I \% aqueous uranyl acetate for $18 \mathrm{hr}$. The pellets were washed in water, dehydrated in tertiary butanol mixtures (Johansen, 1940) and embedded in Araldite. Sections were cut with a diamond knife on a LKB Ultratome and stained with saturated uranyl acetate in $50 \%$ ethanol (Gibbons \& Grimstone, 1960) and then with lead citrate (Reynolds, 1963). The specimens were examined and photographed in a Siemens Elmiskop I or a Zeiss EM 9 at $60 \mathrm{kV}$. 


\section{RESULTS}

Cytochrome analysis. Respiratory-competent cultures of Saccharomyces cerevisiae strains II and I D showed absorption peaks due to cytochrome $a$ at $600 \mathrm{~m} \mu, b$ at 560 and $530 \mathrm{~m} \mu$ and $c$ at 550 and $520 \mathrm{~m} \mu$. All the $\rho$ - strains showed only cytochrome $c$. Spectra from the two $\rho+$ parents and six PEA-induced $\rho-$ clones are reproduced in Fig. I. The I D $\rho$ - and $2 \mathrm{D} \rho-$ spectra have very small maxima at $600 \mathrm{~m} \mu$, equivalent to a cytochrome $a$ content less than $5 \%$ that of the $\rho+$ strains, and none of the $\rho-$

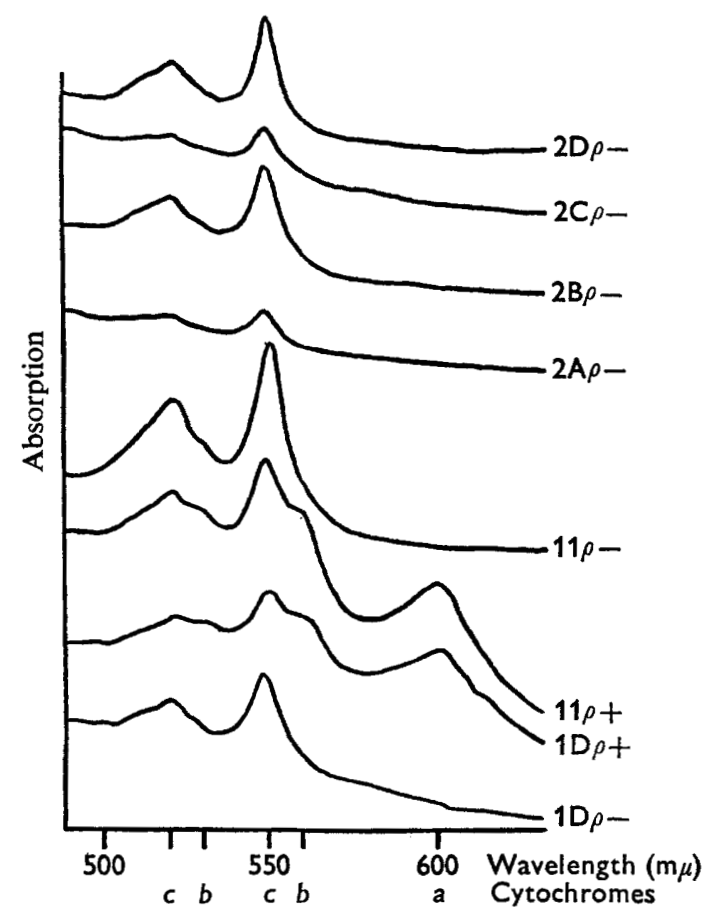

Fig. I. Cytochrome absorption spectra of respiratory-competent $(\rho+)$ and phenethylalcohol-induced petite $(\rho-)$ strains of Saccharomyces cerevisiae.

spectra have the $b$ shoulder at $560 \mathrm{~m} \mu$. The cytochrome $c$ peaks in the $\rho$ - tetrad appear to have segregated $2: 2$ between $2 \mathrm{~A}$ and $2 \mathrm{C}$ (low) and $2 \mathrm{~B}$ and $2 \mathrm{D}$ (high). In the $\rho+$ parent strains, cytochrome $c$ peaks are higher in I I than in I D.

Electron microscopy. We were concerned, in this investigation, with the question whether the petite mutation results in mitochondria which are either visibly aberrant or non-cristate. Mitochondrial profiles were therefore divided into three classes: cristate, non-cristate and aberrant (lamellate or distorted). As the sections were of standard thickness it was possible to compile significant data on the relative frequency of cristae in mitochondrial profiles.

Effect of various mutagens on strain $I D$. In thin sections of respiratory-competent yeasts of Saccharomyces cerevisiae strain I D grown to late logarithmic phase in $2 \%$ 
melibiose, there was great variation in mitochondrial size and shape (Pl. I, fig. I to 4). Cristae were absent from some profiles but were extensively developed in the majority. In a few instances the mitochondria had a lamellate appearance.

The spontaneous petite of strain ID was not distinguishable from the parental strain on the basis of its mitochondrial structure except for somewhat higher numbers of lamellate mitochondria (Pl. I, fig. 5, 6). All other features were the same as the parental type.

Strain I D petites induced with acriflavine had slightly fewer mitochondria in each section than the parent strain (average of two profiles per section as opposed to three per section). Some of the mitochondria were grossly aberrant but none was lamellate. A good proportion of the mitochondria had well-defined cristae (Pl. 2, fig. 7). The rest of the organism was of similar cytology to the parent strain.

Table I. Enumeration of normal and aberrant mitochondria in respiratorycompetent $(\rho+)$ and petite $(\rho-)$ strains of Saccharomyces cerevisiae

\begin{tabular}{|c|c|c|c|c|c|}
\hline Strain & Mutagen & $\begin{array}{c}\text { No. of } \\
\text { mitochondrial } \\
\text { profiles } \\
\text { examined }\end{array}$ & $\begin{array}{c}\% \text { cristate } \\
\text { mitochondrial } \\
\text { profiles } *\end{array}$ & $\begin{array}{l}\% \text { completely } \\
\text { non-cristate } \\
\text { mitochondrial } \\
\text { profiles }\end{array}$ & $\begin{array}{c}\% \text { grossly } \\
\text { aberrant or } \\
\text { lamellate } \\
\text { mitochondrial } \\
\text { profiles }\end{array}$ \\
\hline I D $\rho+$ & - & 84 & 73 & $2 \mathrm{I}$ & 6 \\
\hline I D $\rho-$ & Spontaneous & 66 & 70 & 20 & 10 \\
\hline I D $\rho-$ & Ultraviolet & 37 & 54 & 46 & 0 \\
\hline I $\mathrm{D} \rho-$ & Acriflavine & $5 \mathrm{I}$ & 55 & 37 & 8 \\
\hline I D $\rho-$ & $\begin{array}{l}\text { Phenethyl } \\
\text { alcohol }\end{array}$ & 49 & 55 & 27 & 18 \\
\hline II $\rho+$ & - & IOI & 95 & 5 & 0 \\
\hline II $\rho-$ & $\begin{array}{l}\text { Phenethyl } \\
\text { alcohol }\end{array}$ & 50 & 60 & 40 & 0 \\
\hline $2 \mathrm{~A} \rho-$ & $\begin{array}{l}\text { Phenethyl } \\
\text { alcohol }\end{array}$ & 20 & 65 & 35 & 0 \\
\hline $2 \mathrm{~B} \rho-$ & $\begin{array}{l}\text { Phenethyl } \\
\text { alcohol }\end{array}$ & 40 & 52 & 40 & 8 \\
\hline $2 \mathrm{C} \rho-$ & $\begin{array}{l}\text { Phenethyl } \\
\text { alcohol }\end{array}$ & $3 I$ & 55 & 45 & 0 \\
\hline $2 \mathrm{D} \rho-$ & $\begin{array}{l}\text { Phenethyl } \\
\text { alcohol }\end{array}$ & 15 & 47 & 33 & 20 \\
\hline \multicolumn{6}{|c|}{ bove results } \\
\hline \multirow{2}{*}{\multicolumn{2}{|c|}{$\begin{array}{l}\text { Parental types } \\
\text { Petites }\end{array}$}} & 185 & 85 & I 2 & 3 \\
\hline & Petites & 359 & 58 & 35 & 7 \\
\hline
\end{tabular}

* No assessment was made of reductions in the amount of cristal membranes: a profile showing any internal membrane was counted as cristate.

In the petite of strain I D induced with PEA, mitochondria were well developed, often with extensive cristae (Pl. 2, fig. 8). Lamellate mitochondria were quite frequently observed (PI. 2, fig. 9). An additional feature of these yeasts was a proliferation of the endoplasmic reticulum to yield a discontinuous system throughout the cytoplasm (Pl. 2, fig. Io).

The ultraviolet-induced petite of strain I D (Pl. 2, fig. I I) had poorly defined mitochondrial profiles, but nevertheless some of them were clearly cristate. 
Effect of PEA on various strains of Saccharomyces cerevisiae. Two parent strains of S. cerevisiae were used: I D and II. The appearance of strain I D and its PEA-induced petite have already been described above. A petite strain was induced from II by PEA treatment. Petites were also produced by PEA treatment of an II $\times$ ID ascospore tetrad (2A-2D). The mitochondria of strain II respiratory-competent type were larger and had better developed cristae than strain ID (P1. 2, fig. I2, P1. 3, fig. 13); no lamellate or aberrant mitochondria were observed. Other features of the two strains were identical. The five PEA-induced petite strains from I I and the cross with ID all showed similar mitochondrial morphology (Pl. 3, fig. I4 to I8). The major difference between the petite strains and the parent strains was the reduction in the number of cristae in each mitochondrial profile. Also, as in the other petites, an increased proportion of the mitochondrial profiles showed no cristae. There were a number of grossly aberrant mitochondria in two of these petites (2 B and 2D), but no lamellate ones were observed in any of the strains derived from parent strain II.

An analysis of the mitochondrial profiles found in sections of parental and petite strains of $S$. cerevisiae is given in Table I.

\section{DISCUSSION}

By using a fermentable but non-repressing carbohydrate such as melibiose it has been possible to investigate the mitochondrial structure of Saccharomyces cerevisiae petites without the complication of glucose repression. Melibiose is a disaccharide of glucose and galactose but the concentration of the repressing metabolite apparently does not reach a critical value during fermentation. Although respiration is absent in petite yeasts, glucose repression could still be an important factor by interfering with the biogenesis of the mitochondria.

The mitochondrial cytochromes $a$ and $b$ were absent from petite strains, whatever the means of mutagenesis. This is exactly the situation which occurs when respiratorycompetent yeasts are treated with chloramphenicol, erythromycin and similar antibiotics which are believed to specifically inhibit protein synthesis in the mitochondria (Clark-Walker \& Linnane, I966, 1967; Huang, Biggs, Clark-Walker \& Linnane, 1966).

The petite yeasts show changes in their mitochondrial structure, but as the mitochondria of Saccharomyces cerevisiae are so varied even in the respiratory-competent type, care has to be taken in the interpretation of these changes. For one thing, it is evident that in respiratory-competent strains there is variation in the appearance of mitochondria from one strain to another. Petites produced from these strains show a similar variation in mitochondrial structure. However, it is possible to detect definite trends in the structural changes accompanying the petite mutation (see Table I). In strain I D there was a decrease in the number of mitochondrial profiles with cristae in all the petites except the spontaneous petite. This may mean that the spontaneous petite arose from some minimal change whereas the mutagens caused multiple lesions. Strain I I showed no aberrant or lamellate mitochondrial profiles either in the parental type or in its petite. The reduction in the number of cristate profiles was similar to the strain I $D$ petites. The apparent lack of cristae in a given profile does not necessarily imply that the mitochondrion as a whole is non-cristate, but it shows that there is at least a decrease in the amount of inner membrane. 
These results are in contrast with those of Avers, Pfeffer \& Rancourt (1965) and Yotsuyanagi (1962) who reported extensive loss of cristae and disorganization in the mitochondria of cytoplasmic petites. It may be significant that these two groups both used glucose media thereby permitting glucose repression. Our results are more in agreement with those of Bowers et al. (1967) who avoided repression by using a chemostat with low levels of glucose and found well-defined cristate mitochondria in a tricarboxylic acid mutant of Saccharomyces.

The petites produced from the I $D \times$ I I cross had a similar reduction of cristate profiles to the parent strain petites. A feature of interest here, however, is the $2: 2$ segregation for aberrant mitochondria. This could be evidence of nuclear control over mitochondrial structure, even in the petite condition.

One of the aims of the present investigation was to compare petites produced by PEA with those induced by other mutagens. PEA is known to prevent the replication of bacterial DNA (Lark \& Lark, I966) and a similar interference with replication of mitochondrial DNA may be responsible for PEA-induction of petites. Of the six PEA petites examined, five were indistinguishable from the acriflavine or ultraviolet-induced petites. The sixth, from strain I D, showed a large amount of membrane distributed throughout many of the organisms. Any abnormalities in the PEA-induced petite mitochondria, apart from the reduction in cristal material, do not seem significant when compared with the inherent variation in mitochondrial structure in respiratory competent $(\rho+)$ cells of strain I D. We have not therefore been able to detect any specific effect of PEA on mitochondrial structure, which differs from any of the other mutagens.

Thus it can be concluded that the main result of the change from $\rho+$ to $\rho-$ is a reduction in the amount of mitochondrial cristal membranes. Contrary to previous authors, we do not find any characteristically $\rho$ - abnormality which we would consider reliable enough to be used as a morphological marker for the cytoplasmic inheritance of mitochondrial characters. On the contrary, what mitochondrial peculiarities we have found seem to be under nuclear control.

There are limitations in comparative work on the ultrastructure of petite and normal mitochondria using the methods described in this paper. 'It is hoped, however, to improve the resolution of mitochondrial variation by examining isolated mitochondrial fractions.

One of us (N.G.M.) wishes to thank the Medical Research Council for a postdoctoral fellowship.

\section{REFERENCES}

Avers, C. J., Pfeffer, C. R. \& Rancourt, M. W. (I965). Acriflavine induction of different kinds of 'petite' mitochondrial populations in Saccharomyces cerevisiae. J. Bact. 90, 481.

Avers, C. J., Rancourt, M. W. \& Lin, F. H. (1965). Intracellular mitochondrial diversity in various strains of Saccharomyces cerevisiae. Proc. natn. Acad. Sci. U.S.A. 54, 527.

Bowers, W. D., MCClary, D. O. \& OGUR, M. (1967). Electron microscopy of a tricarboxylic acid mutant of Saccharomyces. J. Bact. 94, 482.

Clark-Walker, G. D. \& Linnane, A. W. (1966). In vivo differentiation of yeast cytoplasmic and mitochondrial protein synthesis with antibiotics. Biochem. biophys. Res, Commun. $25,8$.

Clark-Walker, G. D. \& Linnane, A. W. (1967). The biogenesis of mitochondria in Saccharomyces cerevisiae. A comparison between cytoplasmic respiratory-deficient mutant yeast and chloramphenicol-inhibited wild type cells. J. Cell Biol. 34, I. 
Ephrussi, B., HotTinguer, H. \& ChImenes, A. M. (1949). Action de l'acriflavine sur les levures. I. La mutation 'petite colonie'. Annls Inst. Pasteur, Paris 76, 351.

GibBons, I. R. \& Grimstone, A. V. (1960). On flagellar structure in certain flagellates. J. biophys. biochem. Cytol. 7, 697.

Huang, M., Biggs, D. R., Clark-Walker, G. D. \& Linnane, A. W. (1966). Chloramphenicol inhibition of the formation of particulate mitochondrial enzymes of Saccharomyces cerevisiae. Biochem. biophys. Acta II4, 434.

Johansen, D. A. (1940). Plant Microtechnique, Ist ed. New York: McGraw-Hill.

LARK, K. G. \& LARK, C. (1966). Regulation of chromosome replication in E. coli: a comparison of the effects of phenethyl alcohol with those of amino-acid starvation. J. molec. Biol. $20,9$.

Marchant, R. \& SMith, D. G. (I968). Membranous structures in yeasts. Biol. Rev. 43, 459.

OsUMI, M. \& КАтоH, T. (1967). Oxygen-induced formation of mitochondrial membrane matrix in respiration-deficient yeasts. Jap. Wom. Univ. J. x4, 67.

ReILly, C. \& Sherman, F. (1965). Glucose repression of cytochrome a synthesis in cytochromedeficient mutants of yeasts. Biochim. biophys. Acta $95,640$.

REYNOLDS, E. S. (1963). The use of lead citrate at high $\mathrm{pH}$ as an electron-opaque stain in electron microscopy. J. Cell Biol. r7, 208.

Roodyn, D. B. \& WILKIE, D. (I968). The Biogenesis of Mitochondria. London: Methuen.

WICKERHAM, L. J. (1946). A critical evaluation of the nitrogen assimilation tests commonly used in the classification of yeasts. J. Bact. 57, 293.

Yotsuyanagi, Y. (1962). Etudes sur le chondriome de la levure. II. Chondriomes des mutants a deficience respiratoire. J. Ultrastruct. Res. 7, $14 \mathrm{I}$.

\section{EXPLANATION OF PLATES}

\section{Plate I}

Fig. I to 4. Sections of respiratory-competent cells of Saccharomyces cerevisiae parent strain I D, showing variations in mitochondrial morphology. $\times 47,000 ; 32,000 ; 26,000 ; 54,000$.

Fig. 5, 6. Spontaneous petite of strain ID. Mitochondrial profiles are numerous and range from normal cristate forms to lamellate types. $\times 45,000 ; 50,000$.

\section{Plate 2}

Fig. 7. Acriflavine-induced petite of strain I D, showing some cristate and some non-cristate mitochondrial profiles. $\times 23,000$.

Fig. 8 to 10. Phenethyl alcohol (PEA-)induced petite of strain I D, showing essentially normal cristate mitochondrial profiles (fig. 8), a lamellate profile (fig. 9) and proliferation of endoplasmic reticulum (fig. 10). $\times 40,000 ; 50,000 ; 32,000$.

Fig. II. Ultraviolet-induced petite of strain ID. The mitochondrial profiles are poorly defined, but some are distinctly cristate. $\times 25,000$.

Fig. I2. Respiratory-competent yeast of parent strain II, with regular, well-defined, cristate mitochondrial profiles. $\times 60,000$.

\section{Plate 3}

Fig. 13. Respiratory-competent parent strain II. $\times 49,000$.

Fig. 14. PEA-induced petite of strain II, illustrating reduction in the number of cristae in the mitochondrial profiles. $\times 50,000$.

Fig. 15, I6. PEA-induced petite of strain $2 \mathrm{~B}$ (resulting from II $\times$ I D cross). Some aberrant mitochondria (Fig. I5) and some normal cristate profiles (Fig. I6) are visible. $\times$ 17,000; 72,000.

Fig. 17, 18. PEA-induced petite of strain 2D (resulting from I I $\times$ I D cross), showing aberrant but basically cristate mitochondrial profiles. $\times 45,000 ; 45,000$. 
Journal of General Microbiology, Vol. 56, No. I

Plate I
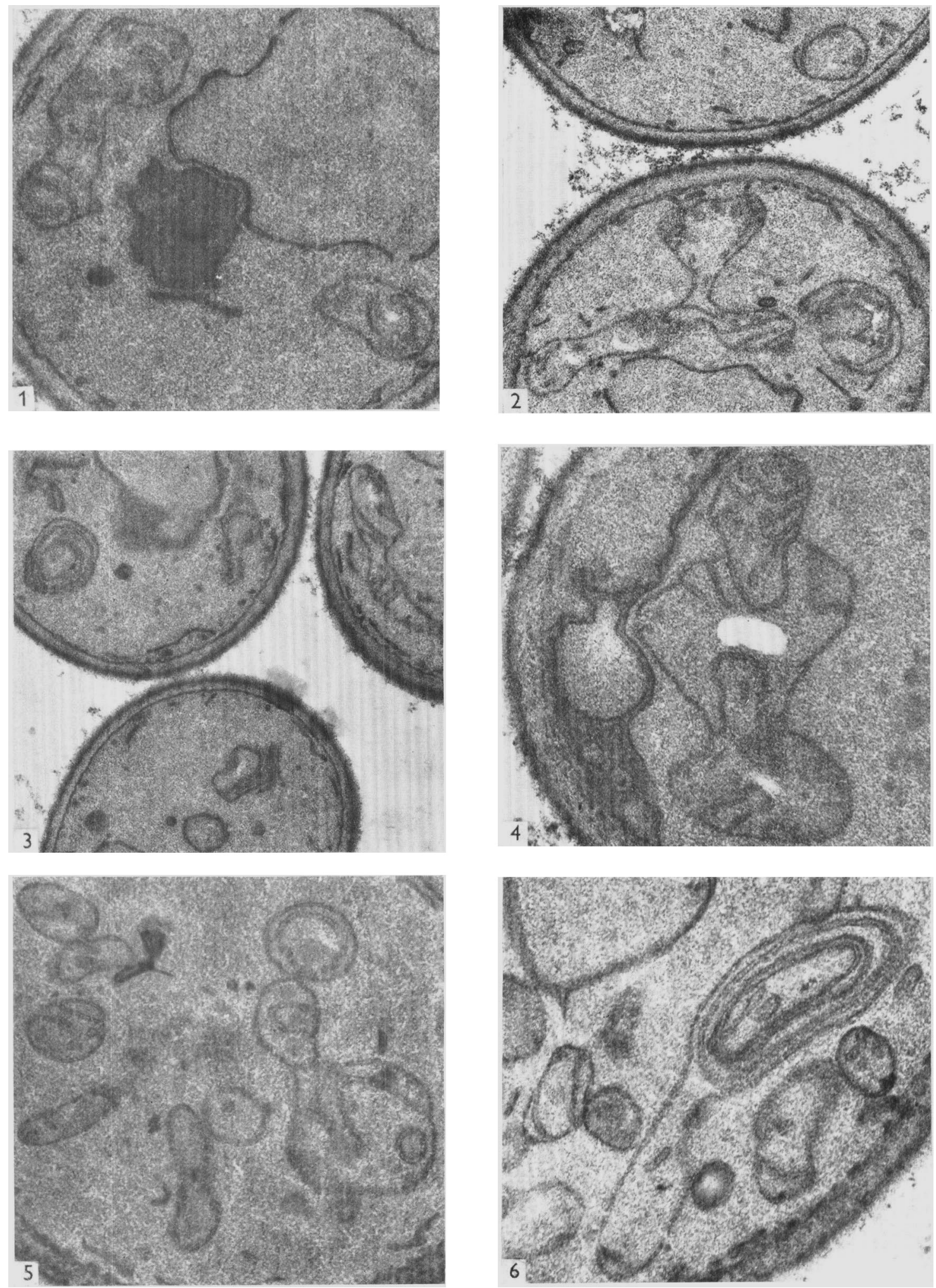

D. G. SMITH AND OTHERS

(Facing p. 54) 
Journal of General Microbiology, Vol. 56, No. I

Plate 2
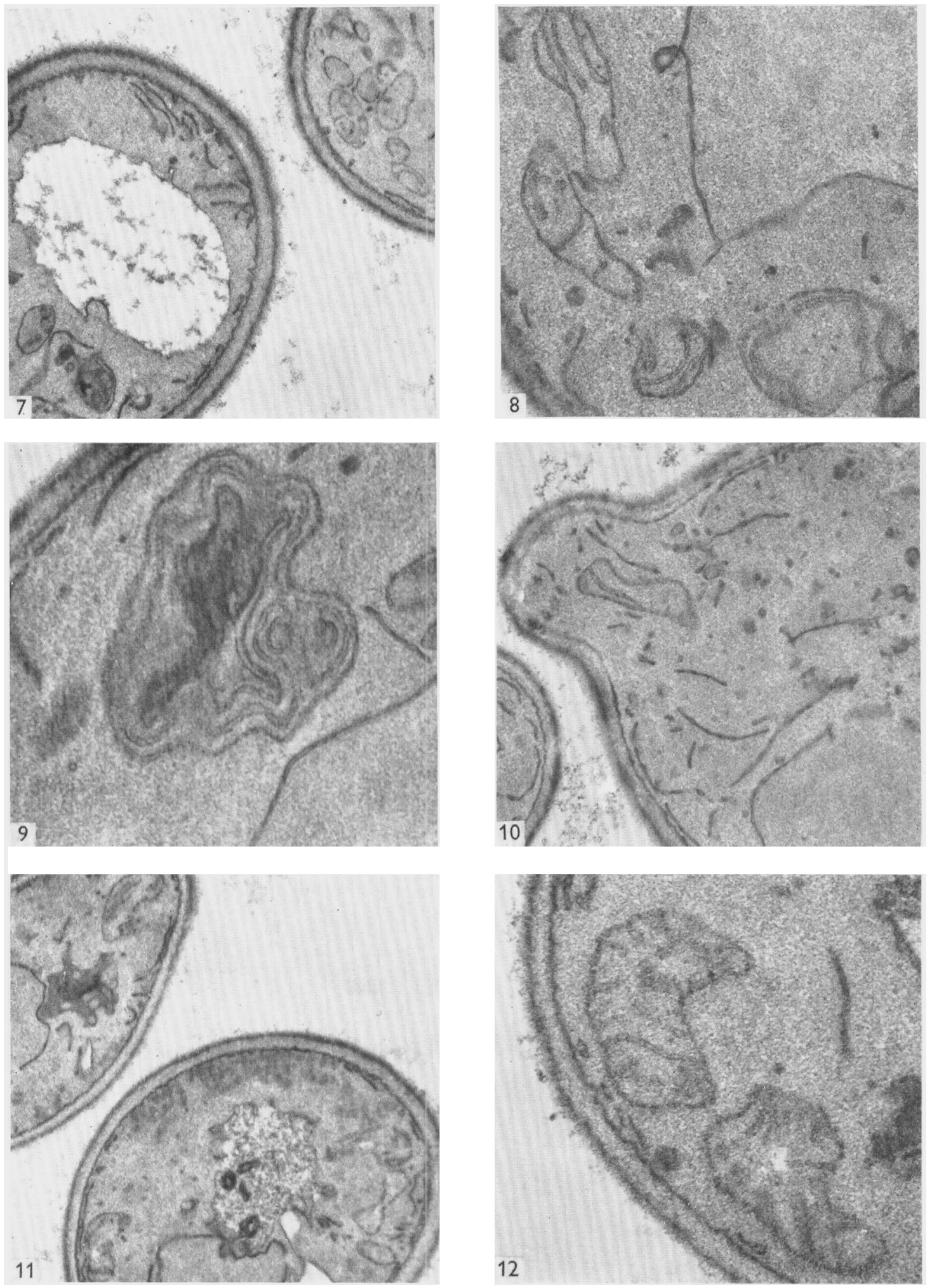

D. G. SMITH AND OTHERS 
Journal of General Microbiology, Vol. 56, No. I

Plate 3
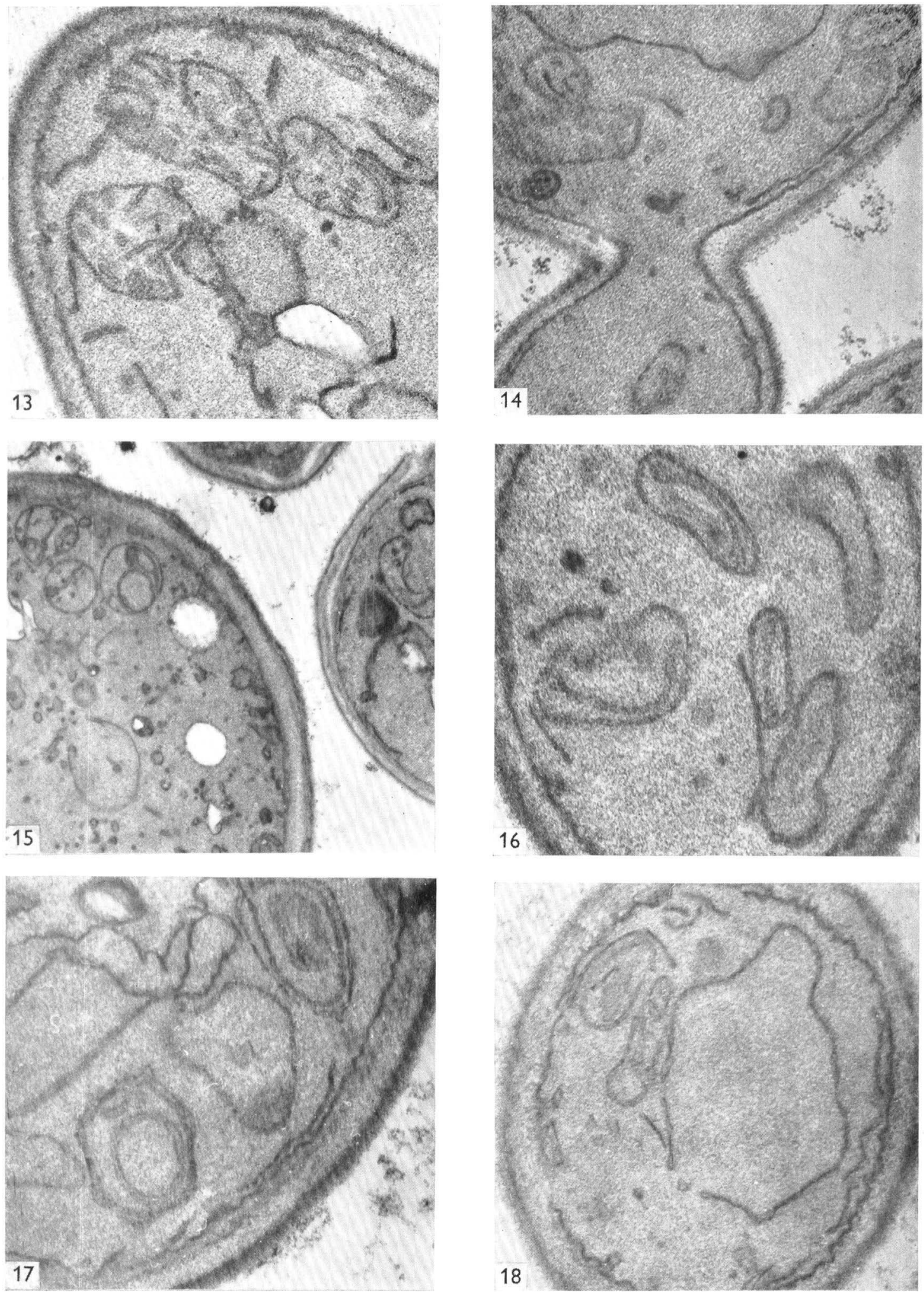

D. G. SMITH AND OTHERS 